\title{
sciendo
}

\section{FEEDING, RESTING AND AGONISTIC BEHAVIOR OF PREGNANT BOER GOATS IN RELATION TO FEEDING SPACE ALLOWANCE}

\author{
Mesut Y1ldırır ${ }^{1,2 \downarrow}$, Gürbüz Daș ${ }^{1,3}$, Christian Lambertz ${ }^{1,4}$, Mathias Gauly ${ }^{1,4}$ \\ ${ }^{1}$ Department of Animal Sciences, Georg-August-University, Göttingen, Germany \\ ${ }^{2}$ Department of Livestock and Aquaculture Research, General Directorate of Agricultural Research and \\ Policy (GDAR), Yenimahalle-Ankara, Turkey \\ ${ }^{3}$ Institute of Nutritional Physiology 'Oskar Kellner', Leibniz Institute for Farm Animal Biology (FBN), \\ Dummerstorf, Germany \\ ${ }^{4}$ Faculty of Science and Technology, Free University of Bozen-Bolzano, Bolzano, Italy \\ •Corresponding author: mesutyildirir@hotmail.com
}

\begin{abstract}
The aim of this study was to test the effect of the animal to feeding place ratio on behavior of meat goats during gestation. In one group ( $F P-1.0 ; n=10)$ every goat had a single feeding place while in the other group (FP- $0.5 ; n=10)$ two goats had to share one feeding place. Behaviors were analyzed with the continuous sampling method for $24 \mathrm{~h}$ for each animal. Social dominance index was used by ranking individuals as low-, medium- and high-ranking according to withdrawal and displacement behaviors. Total daily feeding duration decreased $(\mathrm{P}=0.001)$ from $5.2 \mathrm{~h}$ to $4.2 \mathrm{~h}$ by reducing feeding place. Feeding duration was neither affected by social rank $(\mathrm{P}=\mathbf{0 . 3 6 2})$ nor its interaction with feeding place $(\mathrm{P}=\mathbf{0 . 1 5 9})$. Feeding frequency was higher in FP-0.5 than FP-1.0 $(\mathrm{P}=0.001)$. Lowranking goats had a higher feeding frequency $(\mathrm{P}<0.001)$. Resting duration (lying and standing) decreased in medium- and low-ranking goats in FP-0.5 $(\mathrm{P}=0.001)$ while all rank categories were similar in FP-1.0. Competition behaviors increased $(\mathrm{P}=0.001)$ in medium- and low-ranked goats in FP- 0.5 but were similar between rank categories in FP-1.0. Feeding place and social rank had an effect $(\mathbf{P} \leq \mathbf{0 . 0 0 9})$ on some of the agonistic behaviors. Agonistic interactions such as displacement, butting and ignoring were affected by the interaction of feeding place and social rank $(P \leq 0.005)$. In conclusion, the reduction of the number of feeding places from 1.0 to 0.5 per goat in pregnant hornless Boer goats negatively affected feeding, competition, resting and aggressive behaviors, whereas low-ranking animals suffered more from competition and aggression behaviors.
\end{abstract}

Key words: agonistic behavior, competition, feeding behavior, goats, resting behavior

Feeding management should fulfill the behavioral needs of the animal species by using specific information on the behavioral interactions among individuals. A number of studies examined the impact of competition at the feeding place when increasing the number of animals sharing each 'feed space' on the animal behavior (e.g. 
Corkum et al., 1994; Fregonesi and Leaver, 2002; DeVries et al., 2004; Jørgensen et al., 2007; Ferris et al., 2013). As social animals, ruminants tend to synchronize their feeding behavior and access the feed bunk as a group (Nielsen et al., 2016). A reduction of feeding space allowance from 20 to $10 \mathrm{~cm} /$ head led to a significant reduction in the amount of time spent feeding (Loretz et al., 2004).

The agonistic behavior influences an individual's access to vital resources, such as food, water and shelter (Miranda-de la Lama and Mattiello, 2010). DeVries et al. (2004) documented an increased feeding time and a decreased aggression level when feeding space was increased from 0.5 to $1.0 \mathrm{~m}$ per animal. Similarly, Jørgensen et al. (2007), reported that a decreased number of feeding places per dairy goat increased aggression and it was recommended to raise not more than one goat per feeding place. But, Loretz et al. (2004) did not find any effects of reducing feeding place from 1.0 to 0.5 per head on aggressive interactions in different breeds of goats including Boer goats.

Competition and overstocking affect the resting time of cows in the pen (Fregonesi et al., 2007; Cook, 2019). Increased stocking density increases social stress and aggression and reduces lying time in cows (Krawczel and Lee, 2019). Resting behavior can be negatively influenced by aggression in goats (Aschwanden et al., 2009). In contrast, Jørgensen et al. (2007) reported that the percentage of time spent lying increased when the number of goats per feeding place rose. However, Bøe et al. (2006) showed that reduced lying space resulted in a reduction of lying time and a large increase in the number of displacements in sheep. The proportion of time the goats spent lying decreased significantly with decreasing space allowances (Loretz et al., 2004). In addition, the lowest ranked goats spent less time resting and spent more of their resting time in the low-comfort activity area (Andersen and Bøe, 2007).

Social dominance is the ability of an individual to have priority over others for access to scarce resources (Miranda-de la Lama and Mattiello, 2010). Therefore, social interactions among goats need to be considered to improve husbandry conditions. Barroso et al. (2000) clearly demonstrated the existence of a stable social hierarchy throughout several months and a linear hierarchic order. Body weight and having horns are the most important factors determining the level of aggressive behavior and thus dominance status of an individual (Conway et al., 1996; Barroso et al., 2000; Tölü et al., 2007). High-ranking goats benefit from available resources more than low-ranking goats by spending more time feeding (Andersen and Bøe, 2007; Aschwanden et al., 2009; Loretz et al., 2004; Pascual-Alonso et al., 2013).

The physiological situation can affect the behavior. General activity decreases with the advancement of the gestation in sheep (Averós et al., 2014). We expected that reduced feeding place allowance would potentially increase the aggression and decrease time spent feeding of pregnant meat goats. Negative effects might be exacerbated or lessen through gestation. However, direct comparisons of feeding arrangement on resting behavior for advancing gestation of goats are not available in the literature.

The objective of this study was to determine the effect of reduced feeding place allowance on the feeding, resting, competition and aggression behavior and potential welfare implications of hornless Boer goats during middle gestation. 


\section{Material and methods}

\section{Experimental design}

Two runs with a total of 40 goats were conducted. In each run, goats had either $1.0(\mathrm{FP}-1.0, \mathrm{n}=20)$ or $0.5(\mathrm{FP}-0.5, \mathrm{n}=20)$ feeding places available per head. Each repetition lasted 6 weeks.

\section{Animals and feeding}

All animals were hornless Boer goats reared at the Department of Animal Sciences of the University of Göttingen, Germany were used. The animals were in the second to third month of pregnancy at the start of experiment both of first and the respective repetition. Groups were balanced for initial body weight (BW; 48 to $75 \mathrm{~kg}$ ) and age ( 2 to 8 years of age).

On average, each animal received $2.2 \mathrm{~kg}$ dry matter hay and $0.8 \mathrm{~kg}$ dry matter concentrate per day. The daily amount of hay and concentrate was delivered at two times $(08.00$ and $13.00 \mathrm{~h})$. Hay was refilled after the concentrate was consumed. Water was provided ad libitum.

Animals of each treatment were kept in a single pen sizing $13 \mathrm{~m}^{2}$, and hence providing $1.3 \mathrm{~m}^{2}$ per animal. Pens were bedded with straw and equipped with 10 feeding places that measured $37 \mathrm{~cm}$ in width for FP-1.0, whereas half of them were blinded in the FP- 0.5 treatment by solid wooden wallboards so that 5 feeding places were accessible in the FP-0.5 pen and 10 in the FP-1.0 pen.

\section{Behavioral observations and dominance index}

The goats were individually marked on the back for identification in video records. The animals were video-recorded during the entire experimental period of 6 weeks in both runs. Each pen was monitored with 1 or 2 video cameras (Panasonic, digital 1/3" Standard S/W-Camera Type WV-BP 330, Matsushita Electric Industrial Co. Ltd., Osaka, Japan, 2003) with high resolution (570 line) and sensitivity (0.08 lx F1.4 lens) for infrared. For the night records, the cameras were mounted with additional infrared emitters (LED-Infrarotscheinwerfer $950 \mathrm{~nm}$, Weitflut, 15W, Type WFL-II/LED 15). A digital video recorder (Panasonic, Model AG-TL 750 E) was used for recording and video analysis was performed using the Interact software (Version 13.0, Mangold International $\mathrm{GmbH}$, Arnstorf, Germany). Behavior traits were analyzed with the continuous sampling method over 24 hours of each of the 6 experimental weeks (6th day of the week), resulting in $144 \mathrm{~h}$ of fully evaluated video-records for each animal. The behavior of feeding, resting, competition and agonistic interactions were adopted from Conway et al. (1996), Andersen and Bøe (2007), and Ehrlenbruch et al. (2010) (Table 1). Behaviors were analyzed both as frequency (number of the respective behavior performed per hour) as well as duration (the respective behavior performed in min per hour).

Individual goats within each treatment group were evaluated according to their dominance (D) as suggested by Lamprecht (1986). Withdrawal and displacement behavior of each goat were recorded throughout the trials. All possible comparisons among individuals of a treatment were done, and D was calculated as following: 
$\mathrm{D}=$ number of subdominant individuals / (number of dominant individuals + number of subdominant individuals) x 100. The coefficient ' $D$ ' indicates the percentage of animals dominated in relation to all animals with which the individual has interacted within the treatment. Furthermore, animals of each treatment were classified either as low $(\mathrm{D}<0.33)$, medium $(\mathrm{D}=0.33-0.66)$ or high-ranking $(\mathrm{D}>0.66)$ individuals by using the classifications of Barroso et al. (2000).

Table 1. Definitions of the behaviors to assess impact of number of FP for Boer goats

\begin{tabular}{|c|c|c|}
\hline Behavior & Definition & Recording* \\
\hline Feeding & Head through the feed barrier & $\mathrm{F} \& \mathrm{D}$ \\
\hline \multicolumn{3}{|l|}{ Resting } \\
\hline lying & Lying down in any resting position & $\mathrm{F} \& \mathrm{D}$ \\
\hline standing & Standing without queuing or performing any other behavior & $\mathrm{F} \& \mathrm{D}$ \\
\hline \multicolumn{3}{|l|}{ Competition } \\
\hline attempting & Attempt to reach a feeding place that is occupied & $\mathrm{F} \& \mathrm{D}$ \\
\hline queuing & $\begin{array}{l}\text { Head oriented towards the feed barrier; waiting in front of the } \\
\text { feeder while all feeding places are occupied }\end{array}$ & $\mathrm{F} \& \mathrm{D}$ \\
\hline \multicolumn{3}{|c|}{ Agonistic interaction } \\
\hline fighting & $\begin{array}{l}\text { Number of continuous aggressive interactions (head to head } \\
\text { and pushing each other with physical contact) }\end{array}$ & $\mathrm{F}$ \\
\hline threatening & $\begin{array}{l}\text { Directing the forehead towards the opponent without physical } \\
\text { contact }\end{array}$ & $\mathrm{F}$ \\
\hline withdrawing & $\begin{array}{l}\text { A goat withdraws from another after threatening or physical } \\
\text { contact }\end{array}$ & $\mathrm{F}$ \\
\hline heading & $\begin{array}{l}\text { While resting, continuous butting another goat in the resting } \\
\text { area with the head }\end{array}$ & $\mathrm{F}$ \\
\hline butting & While feeding, continuous butting another goat with the head & $\mathrm{F}$ \\
\hline displacing & $\begin{array}{l}\text { An active behavior that a goat forces another goat to change/ } \\
\text { leave the feeding place }\end{array}$ & $\mathrm{F}$ \\
\hline displaced & $\begin{array}{l}\text { A passive behavior that a goat stops feeding after being forced } \\
\text { by another goat }\end{array}$ & $\mathrm{F}$ \\
\hline ignoring & $\begin{array}{l}\text { While feeding, ignoring another goat/other goats that attempts } \\
\text { to reach the same feeding place }\end{array}$ & $\mathrm{F}$ \\
\hline
\end{tabular}

*Recordings were evaluated either as frequency $(\mathrm{F})$ : total number of the respective behavior performed per hour; or as duration (D): time spent for the respective behavior per hour $(\mathrm{min} / \mathrm{h})$.

\section{Statistical analyses}

All analyses were performed with SAS, version 9.3 (SAS Institute, 2014). Behavioral parameters, quantified hourly as frequency or duration were analyzed with repeated measures analysis of variance using Proc Mixed. The model included the fixed effects of treatment (FP-1.0 and FP-0.5), rank (low, medium and high), hour (1-24), experimental week (1-6) and experimental repetition $(1,2)$. Furthermore, all possible two-way interactions between these factors as well as all possible three-way interactions among triple combinations of factors that included either treatment and/ or rank, were added to the model. Because the observations were made on the same individuals for $24 \mathrm{~h}$ in each of 6 weeks in each experimental repetition, the effect of 
repeatedly observed animal (subject) was also included in the model. The covariance structure was set to be compound symmetry. Post-hoc comparisons were performed with the Tukey test with a significance level of $\mathrm{P}<0.05$.

\section{Results}

\section{Feeding duration and frequency}

Reduced feeding place decreased feeding time, but increased feeding frequency (Table 2). Feeding frequency was the highest in low-ranking goats, without a significant interaction with number of feeding place. Neither the rank order nor the interaction between number of feeding place and rank order had a significant effect on the feeding duration (Table 2).

Table 2. Duration (D; $\mathrm{min} / \mathrm{h})$ and frequency $(\mathrm{F} ;$ count/h) of feeding, resting, competition and agonistic interactions according to FP per goat and social rank categories in hornless Boer goats (Data shown as LSMEANS and SE)

\begin{tabular}{|c|c|c|c|c|c|c|c|c|c|c|}
\hline \multirow{2}{*}{ Behaviors } & \multicolumn{4}{|c|}{ Feeding place (FP) } & \multicolumn{5}{|c|}{ Social Rank (SR) } & \multirow{2}{*}{$\mathrm{FP} \times \mathrm{SR}$} \\
\hline & FP-1 & FP-0.5 & SE & $\mathrm{P} \leq$ & High & Medium & Low & SE & $\mathrm{P} \leq$ & \\
\hline \multicolumn{11}{|l|}{ Feeding } \\
\hline feeding D & 13.0 & 10.5 & 0.20 & 0.001 & 12.0 & 12.1 & 11.2 & 0.26 & 0.362 & 0.159 \\
\hline feeding $\mathrm{F}$ & 1.7 & 3.2 & 0.09 & 0.001 & $2.1 \mathrm{~b}$ & $2.3 \mathrm{~b}$ & $2.9 \mathrm{a}$ & 0.11 & 0.001 & 0.170 \\
\hline \multicolumn{11}{|l|}{ Resting } \\
\hline lying D & 39.4 & 37.7 & 0.27 & 0.113 & 40.1 & 38.1 & 37.5 & 0.35 & 0.079 & 0.003 \\
\hline lying $\mathrm{F}$ & 1.6 & 1.5 & 0.04 & 0.079 & $1.6 \mathrm{ab}$ & $1.4 \mathrm{~b}$ & $1.6 \mathrm{a}$ & 0.05 & 0.039 & 0.001 \\
\hline standing D & 6.1 & 6.9 & 0.15 & 0.080 & 6.2 & 6.3 & 7.2 & 0.18 & 0.195 & 0.361 \\
\hline standing F & 2.1 & 2.0 & 0.09 & 0.871 & $2.0 \mathrm{ab}$ & $1.9 \mathrm{~b}$ & $2.3 \mathrm{a}$ & 0.11 & 0.034 & 0.005 \\
\hline \multicolumn{11}{|l|}{ Competition } \\
\hline attempting D & 0.02 & 0.53 & 0.02 & 0.001 & $0.12 \mathrm{~b}$ & $0.18 \mathrm{~b}$ & $0.52 \mathrm{a}$ & 0.03 & 0.001 & 0.001 \\
\hline attempting $\mathrm{F}$ & 0.1 & 0.8 & 0.04 & 0.001 & $0.3 \mathrm{~b}$ & $0.4 \mathrm{~b}$ & $0.6 \mathrm{a}$ & 0.05 & 0.002 & 0.001 \\
\hline queuing D & 0.04 & 2.10 & 0.06 & 0.001 & $0.3 \mathrm{c}$ & $1.1 \mathrm{~b}$ & $1.9 \mathrm{a}$ & 0.07 & 0.001 & 0.001 \\
\hline queuing F & 0.1 & 1.6 & 0.07 & 0.001 & $0.4 \mathrm{c}$ & $0.8 \mathrm{~b}$ & $1.4 \mathrm{a}$ & 0.09 & 0.001 & 0.001 \\
\hline \multicolumn{11}{|c|}{ Agonistic interaction (all as frequency) } \\
\hline withdraw & 0.4 & 0.6 & 0.05 & 0.016 & $0.1 \mathrm{c}$ & $0.5 \mathrm{~b}$ & $1.0 \mathrm{a}$ & 0.06 & 0.001 & 0.079 \\
\hline displacing & 0.6 & 1.3 & 0.08 & 0.001 & $0.6 \mathrm{~b}$ & $1.1 \mathrm{a}$ & $1.2 \mathrm{a}$ & 0.09 & 0.001 & 0.772 \\
\hline displaced & 0.2 & 1.7 & 0.08 & 0.001 & $0.5 \mathrm{c}$ & $0.9 \mathrm{~b}$ & $1.3 \mathrm{a}$ & 0.09 & 0.001 & 0.001 \\
\hline butting & 0.4 & 1.2 & 0.10 & 0.001 & $1.3 \mathrm{a}$ & $0.7 \mathrm{~b}$ & $0.4 \mathrm{~b}$ & 0.1 & 0.001 & 0.053 \\
\hline ignoring & 0.0 & 0.1 & 0.01 & 0.001 & $0.0 \mathrm{a}$ & $0.1 \mathrm{~b}$ & $0.1 \mathrm{ab}$ & 0.01 & 0.007 & 0.023 \\
\hline fighting & 0.1 & 0.2 & 0.02 & 0.004 & 0.2 & 0.2 & 0.1 & 0.02 & 0.401 & 0.376 \\
\hline threating & 0.2 & 0.1 & 0.05 & 0.368 & $0.3 \mathrm{a}$ & $0.1 \mathrm{~b}$ & $0.0 \mathrm{~b}$ & 0.06 & 0.009 & 0.927 \\
\hline heading & 0.4 & 0.6 & 0.05 & 0.003 & $0.7 \mathrm{a}$ & $0.4 \mathrm{~b}$ & $0.3 \mathrm{~b}$ & 0.06 & 0.002 & 0.191 \\
\hline
\end{tabular}

$\mathrm{a}, \mathrm{b}, \mathrm{c}$ - different letters indicate significant differences among the social ranks $(\mathrm{P} \leq 0.05)$. Differences between FP are indicated with the model P-values. 


\section{Resting duration and frequency}

There was a significant interaction between feeding place and social interaction concerning the lying duration, lying frequency and standing frequency, but not on the standing duration (Table 2). Medium- and low-ranked goats had lower lying durations than high-ranked goats when the number of feeding places was reduced $(\mathrm{P}=0.003)$, while goats with different social ranks had similar lying durations in FP-1.0 (Figure 1). Moreover, high-ranked goats in FP-0.5 showed a similar trend of lying duration compared with FP-1.0 (Figure 1). While high-ranking goats had lower $(\mathrm{P} \leq 0.005)$ standing and lying frequency compared with medium- and low-ranking goats in in FP-1.0, these frequencies were similar between social ranks in the group of FP-0.5. There were significant effects of the day on standing and lying duration $(\mathrm{P} \leq 0.05)$, but no stable pattern could be observed with advancing pregnancy.

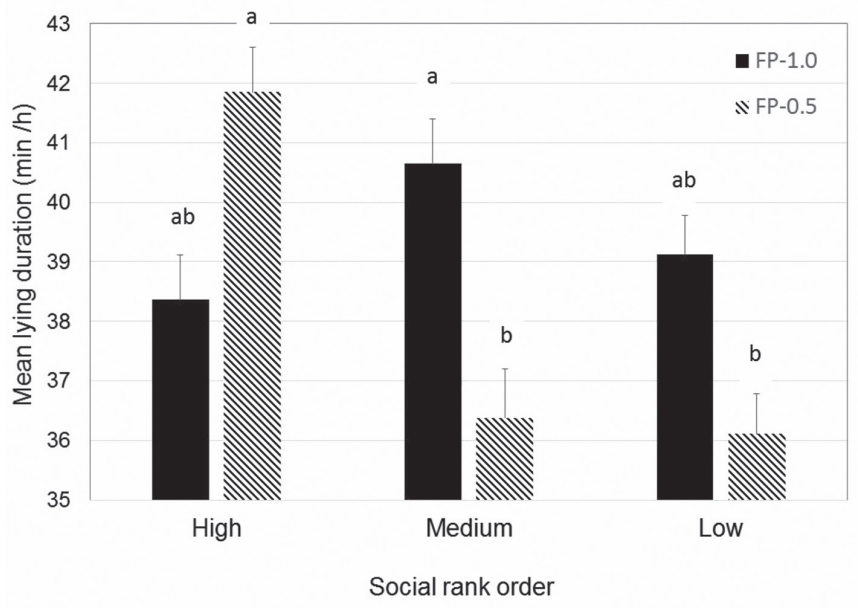

Figure 1. Mean lying behavior with access to 1.0 (FP-1) or 0.5 (FP-0.5) feeding place per goat and the rank interaction. Error bars represent standard errors of LSMEANS, Tukey $\mathrm{P} \leq 0.05$, after a significant $(\mathrm{P} \leq 0.05)$ interaction effect between treatments

\section{Competition duration and frequency}

The duration and frequency of competition behaviors at the feeding place were affected by the interactions between feeding place number and social rank (Table 2). Thereby, rank categories did not differ in FP-1.0 ( $\mathrm{P}>0.05)$ while in FP-0.5 the frequency and duration of competition behaviors increased with decreasing rank category $(\mathrm{P} \leq 0.001)$.

\section{Agonistic interactions}

Feeding place had significant effects on withdrawal, displacing, butting, fighting and heading behaviors, and the social rank category on withdrawal, displacing, butting, threatening and heading behaviors, but there was no interaction between both effects observed (Table 2). Active aggression behaviors (butting, threatening and heading) increased while passive behaviors (withdrawing and displacing) were re- 
duced with decreasing rank category $(\mathrm{P} \leq 0.01)$. Lower-ranking goats in FP-0.5 were exposed to more (rank-feeding place interaction, $\mathrm{P} \leq 0.05$ ) displacement, ignoring and butting behaviors while all rank orders were similar in FP-1.0. The interactions between hour and feeding place number affected all agonistic interactions $(\mathrm{P}<0.001)$. The observed distribution of heading, butting, fighting and threatening are illustrated over a $24 \mathrm{~h}$ period in Figure 2.

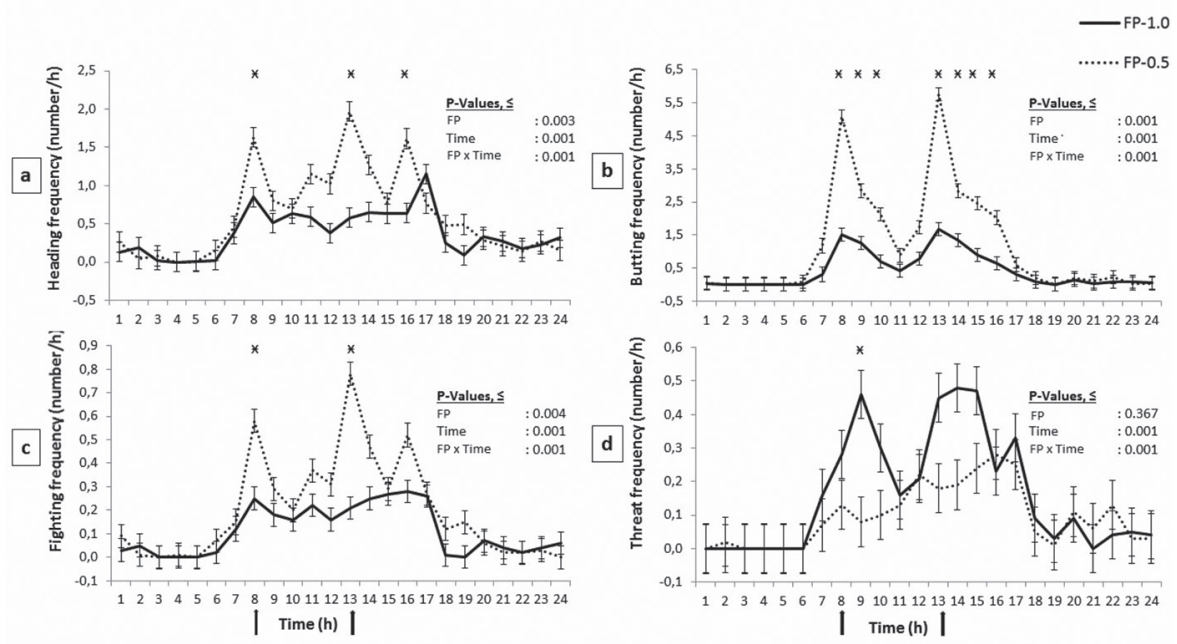

Figure 2. Frequency of agonistic interactions (a - heading, b - butting, $c$ - fighting, $d$ - threat) over a $24 \mathrm{~h}$ period with access to 1 or 0.5 feeding place per goat. Error bars represent standard errors of LSMEANS. P-values shown on the figures refer to interaction effect between treatment (FP) and time. At each time point, LSMEANS accompanied by an asterisk $(*)$ indicate a significant difference, Tukey $\mathrm{P} \leq 0.05$, after a significant $(\mathrm{P} \leq 0.05)$ interaction effect between treatments. Arrows indicate time of feeding at 08.00 and 13.00

\section{Discussion}

This study investigated whether reducing the feeding place to animal ratio from 1.0 to 0.5 affects behaviors such as feeding, resting, competition and agonistic interaction in meat goats during middle of gestation.

Feeding and resting behavior had the largest share of the daytime and there was a large variation in aggressive, feeding and resting behaviors. The feeding time of dairy goats varies between 6 and $9 \mathrm{~h}$ per day in intensive production systems (Abijaoude et al., 2000). Time spent feeding decreased from 5.2 to 4.1 and 3.0 for one, two and three goats per feeding place, respectively, when hay was offered (Jørgensen 
et al., 2007). In this study, the total daily feeding time decreased from $5.2 \mathrm{~h}$ to $4.2 \mathrm{~h}$ by reducing feeding place $(\mathrm{P}=0.0001)$. A significant decrease in time spent feeding was reported in several studies when the feeding places to animals ratio was reduced in cattle (DeVries et al., 2004), pigs (Rasmussen et al., 2006) and sheep (Bøe et al., 2006). Loretz et al. (2004) and Jørgensen et al. (2007) reported a decrease in feeding time when feeding place was restricted in different dairy breeds and Boer goats. Restriction of access to feed by space or time constraints led cows to modify their time budgets and increase their rates of intake (Ferris et al., 2013). A similar effect was reported by Corkum et al. (1994), who observed that goats were able to compensate for shorter times spent at the feeder by increasing the rate of intake. In wide agreement, our results showed that reduced feeding space lowered the duration of feeding but increased its frequency. However, goats may be able to maintain their daily intakes by increasing their mean daily intake rates.

In agreement with the findings of previous studies (Andersen and Bøe, 2007; Aschwanden et al., 2009; Loretz et al., 2004; Pascual-Alonso et al., 2013) highranking goats spend more time for feeding than low-ranking goats. In our study, lowranked goats were frequently forced to leave the feeding place, but feeding duration was similar between rank categories. In addition, the frequency of feeding in FP-0.5 was twice as high as in FP-1.0, independent of the social rank. Harb et al. (1985) showed that submissive cows increased their rate of eating to a greater extent than dominant animals. Confirming these findings low-ranking goats may compensate their intake rates and increase frequency of feeding.

Resting behaviors can be negatively influenced by social conflicts in goats (Aschwanden et al., 2009). Contrarily, Jørgensen et al. (2007) found that increasing the number of goats per feeding place increased the time spent lying. Medium- and low-ranking goats showed less lying duration than high-ranking goats when feeding place was reduced.

Competition and aggressive interactions were increased by restricting feeding place as shown in earlier studies (Fregonesi and Leaver, 2002; Jørgensen et al., 2007; Bøe et al., 2006). In contrast, Loretz et al. (2004) reported that aggressive interactions at the feed barrier were not significantly affected by the number of feeding places available. According to Aschwanden et al. (2009), behavioral reactions without physical contact are more frequent in horned goats than hornless goats. Additionally, avoidance behavior by lower-ranking goats was only rarely seen in the hornless group. In this study, threatening and fighting had the lowest frequency, but displaced, butting and heading were the most encountered aggressive behaviors. Higher-ranking animals constantly reinforce their status by means of continuous aggression (Barroso et al., 2000). Loretz et al. (2004) stated that high-ranking goats often defended several feeding places at once while low-ranking goats had to share feeding places. Increasing competition at the feeding place can be considered as the main reason for increasing aggressive physical contact. However, high-ranking animals did not need physical confrontation to establish a hierarchy.

The decline in activity is one of the expected behavioral changes during pregnancy (Averós et al., 2014). Vas et al. (2013) reported that aggressive behavior decreased during pregnancy. However, in this study there was no significant difference relative 
to stage of pregnancy. Direct comparisons of changing aggression behavior for advancing gestation of these animal types are contradictory and warrant further studies.

In conclusion, competition and aggression increased when feeding place to animal ratio was reduced, especially in lower-ranking goats. In addition, feeding and resting behaviors were negatively affected. Therefore, it is recommended to provide one feeding place for meat goats during middle gestation to prevent agonistic interactions.

\section{Acknowledgements}

We thank Prof. Dr. Wolfgang Holtz, Birgit Sohnrey and Erwin Tönges for their valuable support to conduct this study.

\section{References}

Abija oude J.A., Morand-Fehr P., Tessier J., Schmidely P.H., Sauvant D. (2000). Diet effect on the daily feeding behavior, frequency and characteristics of meals in dairy goats. Livest. Prod. Sci., 64: 29-37.

A n d e r s e n I.L., B ø e K.E. (2007). Resting pattern and social interactions in goats - the impact of size and organisation of lying space. Appl. Anim. Behav. Sci., 108: 89-103.

A s chwanden J., Gygax L., We chs le r B., Ke il N.M. (2009). Loose housing of small goat groups: Influence of visual cover and elevated levels on feeding, resting and agonistic behavior. Appl. Anim. Behav. Sci., 119: 171-179.

Averós X., Lorea A., de Heredia I.B., Arranz J., Ruiz R. (2014). Space availability in confined sheep during pregnancy, effects in movement patterns and use of space. PLoS One, 9: e94767; doi:10.1371/journal.pone.0094767.

B a rro s o F.G., A l a d o s C.L., B o z a J. (2000). Social hierarchy in the domestic goat: effect on food habits and production. Appl. Anim. Behav. Sci., 69: 35-53.

B ø e K.E., B e r g S., A n d e r s e n I.L. (2006). Resting behavior and displacements in ewes - effects of reduced lying space and pen shape. Appl. Anim. Behav. Sci., 98: 249-259.

C o n w a y M.L.T., B l a c k s h a w J.K., D a n i e l R.C.W. (1996). The effects of agonistic behavior and nutritional stress on both the success of pregnancy and various plasma constituents in Angora goats. Appl. Anim. Behav. Sci., 48: 1-13.

C o o k N.B. (2019). Optimizing resting behavior in lactating dairy cows through freestall design. Veterinary Clinics of North America - Food Animal Practice, 35: 93-109; 10.1016/j.cvfa.2018.10.005.

Corkum M.J., B at e L.A., Tenness en T., Liret te A. (1994). Consequences of reduction of number of individual feeders on feeding behavior and stress level of feedlot steers. Appl. Anim. Behav. Sci., 41: 27-35.

De Vries T.J., Von Keys erlingk M.A.G., Weary D.M. (2004). Effect of feeding space on the inter-cow distance, aggression, and feeding behaviour of free-stall housed lactating dairy cows. J. Dairy Sci., 87: 1432-1438.

Ehrlenbruch R., Jørgensen G.H.M., Andersen I.L., Bøe K.E. (2010). Provision of additional walls in the resting area - the effects on resting behavior and social interactions in goats. Appl. Anim. Behav. Sci., 122: 35-40.

F erris C.P., P atters on D.C., Mayn e C.S., O 'C on nel1 N.E. (2013). Effect of feed space allowance and period of access to food on dairy cow performance. Grass and Forage Sci., 70; 59-70.

F r e g o n e s i H.A., L e a v e r J.D. (2002). Influence of space allowance and milk yield level on behavior, performance and health of dairy cows housed in straw yard and cubicle systems. Livest. Prod. Sci., 78: 245-257.

Fregones i J.A., Tu cker C.B., We a ry D.M. (2007). Overstocking reduces lying time in dairy cows. J. Dairy Sci., 90: 3349-3354. 
H a r b M.Y., Vi v i e n S.R., C a m p l ing R.C. (1985). Eating behaviour, social dominance and voluntary intake of silage in group-fed milking cattle. Grass and Forage Sci., 40: 113-118.

$\mathrm{J} ø \mathrm{r} g$ e $\mathrm{n}$ s e n G.H.M., A n d e r s e n I.L., B ø e K.E. (2007). Feed intake and social interactions in dairy goats - the effects of feeding space and type of roughage. Appl. Anim. Behav. Sci., 107: 239-251.

Krawczel P., Lee A. (2019). Lying time and its importance to the dairy cow impact of stocking density and time budget stresses. Vet. Clin. North Am. Food Anim. Pract., 35: 47-60.

L a m precht J. (1986). Structure and causation of the dominance hierarchy in a flock of bar-headed geese (Anser indicus). Behavior, 96: 28-48.

L or e tz C., We chsler B., Ha u s e r R., R ü s ch P. (2004). A comparison of space requirements of horned and hornless goats at the feed barrier and in the lying area. Appl. Anim. Behav. Sci., 87: $275-283$.

Miranda-de la Lam a G.C., Mattiello S. (2010). The importance of social behavior for goat welfare in livestock farming. Small Rumin. Res., 90: 1-10.

Ni els e n B.L., d e J on g I.C., D e Vrie s T.J. (2016). The use of feeding behaviour in the assessment of animal welfare. In: Nutrition and the Welfare of Farm Animals, Phillips C. (ed.). Animal Welfare, vol 16. Springer, Cham.

Pascual-Alonso M., María G.A., Sepúlveda W.S., Villarroel M., Aguayo-U1lo a L., Ga lindo F., M iranda-de 1 a L a ma G.C. (2013). Identity profiles based on social strategies, morphology, physiology, and cognitive abilities in goats. J. Vet. Behav., 8: 458- 465.

R a s m u s s e n D.K., We b e r R., We c h s l e r B. (2006). Effects of animal/feeding-place ratio on the behaviour and performance of fattening pigs fed via sensor-controlled liquid feeding. Appl. Anim. Behav. Sci., 98: 45-53.

SAS Institute Inc., (2014). SAS OnlineDoc $\AA$ Version 9.1.3. Cary, NC, USA.

Tölü C., S avaş T., Pala A., Thomsen H. (2007). Effects of goat social rank on kid gender. Czech J. Anim. Sci., 52: 77-82.

Vas J., Choj n a c k i R., K jør en M.F., Lyng w a C., A nd e r s en I.L. (2013). Social interactions, cortisol and reproductive success of domestic goats (Capra hircus) subjected to different animal densities during pregnancy. Appl. Anim. Behav. Sci., 147: 117-126.

Received: 7 XII 2018

Accepted: 11 IV 2019 